\title{
SUPERVIVENCIA Y CRECIMIENTO DE MACHOS ADULTOS DEL CAMARÓN DE RÍO Cryphiops caementarius Molina, 1782 (CRUSTACEA, PALAEMONIDAE) EXPUESTOS A SALINIDADES
}

\section{SURVIVAL AND GROWTH OF ADULT MALES OF RIVER SHRIMP Cryphiops caementarius Molina, 1782 (CRUSTACEA, PALAEMONIDAE) EXPOSED TO SALINITIES}

\author{
Christian Escobar Gi1 ${ }^{1}$, Marlon Pachamoro Leytón ${ }^{2}$ y Walter Reyes Avalos ${ }^{3}$
}

\begin{abstract}
Resumen
El objetivo fue evaluar la supervivencia y el crecimiento de machos adultos del camarón de río Cryphiops caementarius expuestos a salinidades. El experimento se realizó en 12 acuarios con 501 de agua, cada uno con filtro biológico y con cinco refugios. Las salinidades experimentales fueron 0, 2, 4 y 8\%, implementadas por triplicado. En cada acuario se sembró al azar cinco camarones. Un total de 60 camarones machos adultos $(5.94 \pm 0.11 \mathrm{~cm}$ de longitud total y $8.15 \pm 0.810 \mathrm{~g}$ de peso húmedo) fueron usados en el experimento que duró 60 días. Los camarones expuestos en agua con $4 \%$ de salinidad presentaron alta y significativa $(\mathrm{p}<0.05)$ supervivencia $(>80 \%)$, en cambio en $0 \%$ o y $2 \%$ la supervivencia fue baja (20\%) debido al canibalismo y en $8 \%$ la supervivencia fue muy baja (6\%) debido al estrés osmótico. El crecimiento de los camarones expuestos en salinidad de 4\%o no mostró diferencia significativa ( $\mathrm{p}>0.05$ ) con aquellos expuestos en $0 \%$ y en $2 \%$ de salinidad durante el período de estudio, pero los mayores valores de crecimiento en peso se presentaron en $4 \%$ de salinidad.
\end{abstract}

Palabras clave: estrés osmótico, crecimiento, osmorregulación, supervivencia, Cryphiops.

\begin{abstract}
The aim was to evaluate the survival and growth of adult males of the freshwater shrimp Cryphiops caementarius exposed to salinities. The experiment was carried out in 12 aquariums with 501 of water, each with biological filter and five shelters. The experimental salinities were $0,2,4$ and $8 \%$, implemented in triplicate. Five shrimps were stocked at random in each aquarium. A total of 60 adult male shrimps $(5.94 \pm 0.11 \mathrm{~cm}$ of total length and $8.15 \pm 0.810 \mathrm{~g}$ of wet weight) were used in the experiment that lasted 60 days. Shrimps exposed to water with $4 \%$ of salinity presented high and significant $(\mathrm{p}<0.05)$ survival $(>80 \%)$, whereas in $0 \%$ and $2 \%$ o the survival was low $(20 \%)$ due to cannibalism, and in $8 \%$ the survival was very low $(6 \%)$ due to osmotic stress. Growth of shrimps exposed to $4 \%$ salinity showed no significant difference $(\mathrm{p}>0.05)$ with those exposed to $0 \%$ and $2 \%$ of salinity during the study period, but the highest values of growth in weight were presented to $4 \%$ salinity.
\end{abstract}

Key words: osmotic stress, growth, osmoregulation, survival, Cryphiops.

\section{Introducción.}

El camarón de río Cryphiops caementarius (Molina, 1782) se distribuye desde Lambayeque en Perú hasta Valparaíso en Chile (Moscoso, 2012), y posee alta densidad poblacional solo en los ríos Ocoña, MajesCamaná y Tambo en Arequipa-Perú, desde donde se abasteció al mercado de Lima con 1042 t en el 2015 (Wasiw \& Yépez, 2015; PRODUCE, 2016), y es la especie prioritaria para el biocomercio en Perú (Lleellish et al., 2005). Sin embargo, el cultivo comercial no se ha establecido porque aún faltan solucionar los problemas de interacción y canibalismo entre congéneres que sucede en agua dulce y que afectan la supervivencia, el crecimiento y la producción (Ponce, 1997; Reyes et al., 2006; Reyes, 2012).

En el ambiente natural, C. caementarius transita entre aguas dulces y salobres en su migración como organismo anfídromo (Bauer, 2013), donde la salinidad es uno de los factores ambientales que influye en la distribución del camarón de río durante su desarrollo ontogenético. Durante el verano austral, los camarones hembras y machos migran hacia las parte bajas de los ríos donde se produce el apareamiento, y las hembras incuban los huevos hasta que liberan larvas, las mismas que requieren de agua salobre para continuar con su desarrollo (Viacava et al., 1978; Guerra et al., 1987), de ahí que mayor cantidad de hembras se encuentran 
cerca de las desembocaduras de los ríos porque toleran estrés osmótico. Las postlarvas crecen y resisten salinidades de hasta $24 \%$, pero crecen mejor a $12 \%$, demostrando con ello su gran capacidad osmorreguladora (Reyes et al., 2006), pero la salinidad es el estímulo para la migración hacia el agua dulce (Viacava et al., 1978; Bauer, 2013). Los camarones machos adultos, en cambio, que se encuentran río arriba y en agua completamente dulce, llegan cerca de la desembocadura, debido al incremento del caudal del agua, solo para apareamientos y por ello es probable que se encuentren en ambientes con baja concentración de salinidad. En Macrobrachium rosenbergii, los sub adultos se encuentran en zonas estuarinas y están adaptados a variaciones constantes de salinidad (Chand et al., 2015).

En crustáceos de agua dulce, la osmorregulación es un proceso que mantiene la concentración interna de solutos de manera independiente, de acuerdo a las variaciones de salinidad de medio externo y para ello se requiere gasto de energía (Hill, 2007). Los adultos de $M$. tenellum y $M$. acanthurus son hiperreguladores en bajas salinidades $(<10 \%)$ e hipoconformadores en altas salinidades, lo cual se asocia a organismos de aguas salobres (Signoret et al., 1997). Los adultos de $M$. amazonicum hiperosmorregulan a 20\%o (Belli et al., 2009), en cambio los adultos de $M$. rosenbergii son hiperreguladores hasta en $14 \%$ y osmoconformadores entre $14.5 \%$ a $21 \%$ de salinidad como estrategia para disminuir el gradiente osmótico entre la hemolinfa y el medio externo (Cheng et al., 2003; Huong et al., 2010) y en éstas salinidades hay expresión de genes en las branquias que permiten tolerar dichas salinidades (Barman et al., 2012). Sin embargo, no se conoce la osmorregulación de ningún estado ontogenético de $C$. caementarius, pero dado su comportamiento migratorio de los machos adultos, deben poseer mecanismos osmorregulatorios para soportar cierta concentración de salinidad, cuando en algún momento de su vida se encuentren en contacto con agua salobre, similar a lo sucedido en hembras de la especie, que incrementan los hemocitos y la concentración de oxihemocianina, con el incremento de la salinidad del medio a $6 \%$ y hasta 12\%o (Valverde et al., 2014). En este sentido, los camarones machos de C. caementarius deben soportar cierto estrés osmótico aunque menor que las hembras.

En algunas especies de carídeos las bajas salinidades no afectan la supervivencia pero favorecen el crecimiento. En Atya scabra los adultos toleran el estrés osmótico, durante corto período, entre 5\% y $15 \%$ de salinidad sin causar mortalidad (Rodríguez et al., 1991). En M. rosenbergii la salinidad de $15 \%$ ocasiona alta supervivencia (95\%) (Huong et al., 2010). En M. tenellum la salinidad de 5\% mejora el crecimiento en peso y mantiene alta supervivencia (85\%) (Vega-Villasante et al., 2011).

En el presente trabajo se prueba la hipótesis de que el agua con cierta concentración de salinidad ocasiona, en camarones machos adultos de C. caementarius, inhibición de la interacción y el canibalismo, y mayor crecimiento, similar a lo que sucede en postlarvas de la misma especie durante largo período de exposición salina (Reyes et al., 2006); en cambio, elevadas salinidades afectan la supervivencia por estrés osmótico.

En consecuencia, de acuerdo a los antecedentes con camarones de agua dulce, el objetivo fue evaluar la supervivencia y el crecimiento de machos adultos del camarón de río C. caementarius expuestos a salinidades.

\section{Materiales y métodos. \\ Material biológico.}

Se capturaron 108 camarones del río Pativilca (0907'70''S - 78'34'20''O) a 352 msnm (Provincia de Barranca, Distrito de Pativilca, Lima, Perú) (Figura 1). Los camarones se introdujeron individualmente en vasos de plástico de $200 \mathrm{ml}$ (los vasos tuvieron agujeros para permitir el flujo de agua) y se acondicionaron en una caja de plástico $(0.60 \times 0.40 \times 0.35 \mathrm{~m}$, con $361 \mathrm{de}$ volumen efectivo) con agua del mismo río y con aireación intermitente. La densidad fue de 3 camarones por litro. El transporte terrestre duró $5 \mathrm{~h}$ y no hubo mortalidad. En el laboratorio los camarones se aclimataron por seis días en el mismo sistema de transporte y se alimentaron a partir del tercer día de aclimatación.

Sistema de experimentación.

Se emplearon 12 acuarios $(0.60 \mathrm{~m}$ de largo; $0.31 \mathrm{~m}$ de ancho y $0.35 \mathrm{~m}$ de alto, con área de $0.186 \mathrm{~m}^{2} \mathrm{y}$ volumen efectivo de 55 1), con cinco refugios (tubos de PVC de $5 \mathrm{~cm}$ de diámetro y $10 \mathrm{~cm}$ de longitud) por acuario. En cada acuario se instaló un filtro biológico percolador (2.5 1) con lecho filtrante en dos capas

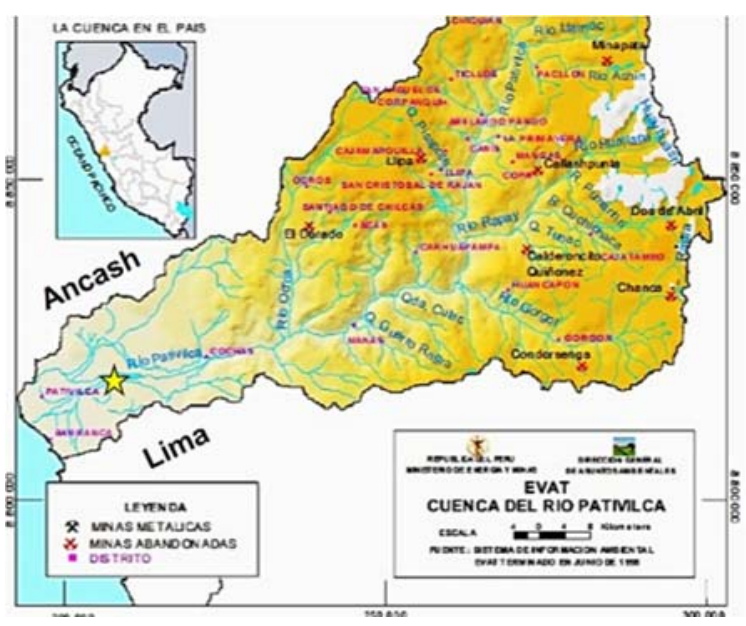

Figura 1. Cuenca del río Pativilca. El símbolo de estrella indica el lugar de captura de camarones de río, cerca del centro Poblado Huayto (Barranca, Lima, Perú). 
constituidos por conchuela triturada y grava, en iguales proporciones.

Salinidades experimentales.

La salinidad de cada tratamiento fue de 0, 2, 4 y $8 \%$, y fue por triplicado. El agua salobre de cada tratamiento se obtuvo por mezcla de agua de mar y agua dulce. El agua de mar (35\%) procedió de la playa Anconcillo (Nuevo Chimbote) y en laboratorio se decantó por dos días y el sobrenadante se filtró con tamiz $(20 \mu \mathrm{m})$ y se guardó en recipientes con tapa para uso posterior. El agua potable fue aireada por $48 \mathrm{~h}$ para eliminar el cloro. Selección, aclimatación y siembra.

En el laboratorio, la especie de camarón fue reconocida según la clave taxonómica de Méndez (1981) y el sexo de los camarones machos se verificó por la presencia de gonóporos en los coxopoditos del quinto par de periópodos. Luego, 60 camarones machos adultos $(5.94 \pm 0.11 \mathrm{~cm}$ de longitud total y $8.15 \pm 0.81$ $\mathrm{g}$ de peso húmedo) en estados de intermuda $\mathrm{C} \mathrm{y}$ de premuda $\mathrm{D}_{1}$ (Reyes \& Lujan, 2003) se seleccionaron al azar. La aclimatación de los camarones a cada salinidad experimental fue por aumento de $2 \%$ cada $12 \mathrm{~h}$ durante $36 \mathrm{~h}$. La salinidad se midió con un refractómetro VEE GEE A366ATC ( $\pm 1 \%$ ). En cada acuario se sembraron al azar cinco camarones (27 camarones $\mathrm{m}^{-2}$ ).

Dieta.

La dieta se elaboró de acuerdo a la formulación de Reyes (2012) y se suplementó con 3\% de levadura Saccharomyces cerevisiae (Cornejo et al., 2015). La composición proximal de la dieta $(30 \%$ de proteína total, $8.1 \%$ de lípidos y $4.6 \%$ de fibra, con $2600 \mathrm{kcal} \mathrm{g}^{-}$ $\left.{ }^{1}\right)$ se calculó según Pezzato (1996). Los gránulos de alimento tuvieron $3 \mathrm{~mm}$ de diámetro. La ración diaria $(08: 00$ y $18: 00 \mathrm{~h})$ fue del $4 \%$ de la biomasa, reajustándose cada 15 días.

Supervivencia y crecimiento.

Los muestreos de toda la población sembrada se realizó cada quince días y durante dos meses. La longitud total (escotadura post orbital - extremo posterior del telson) fue medida con vernier $( \pm 0.1 \mathrm{~mm})$ y el peso con balanza digital portátil TANITA $( \pm 0.1 \mathrm{~g})$. Con los datos se determinaron los parámetros de crecimiento (El-Sherif \& Ali, 2009) y la supervivencia se determinó por conteo directo.

$$
\begin{gathered}
\text { Crecimiento absoluto (CA): } \\
\text { CA }=\mathrm{X} 2-\mathrm{X} 1
\end{gathered}
$$

Ganancia porcentual (GP):

$$
\mathrm{GP}=(\mathrm{X} 2-\mathrm{X} 1 / \mathrm{X} 1) \times 100
$$

Tasa de crecimiento absoluta (TCA):

$$
\mathrm{TCA}=\mathrm{X} 2-\mathrm{X} 1 / \mathrm{t} 2-\mathrm{t} 1
$$

Tasa de crecimiento específica (TCE):

$$
\mathrm{TCE}=[\ln \mathrm{X} 2-\ln \mathrm{X} 1) / \mathrm{t} 2-\mathrm{t} 1] \times 100)
$$

$$
\text { Supervivencia }(\%)=\mathrm{Ni} \times 100 / \mathrm{No}
$$

Donde $\mathrm{X}_{1}$ y $\mathrm{X}_{2}$ es longitud o peso ( $\mathrm{cm} \mathrm{o} \mathrm{g}$ ), inicial $\mathrm{y}$ final; $\mathrm{t}_{1} \mathrm{y} \mathrm{t}_{2}$ es la duración en días; $\ln \mathrm{X}_{1} \mathrm{y} \ln \mathrm{X}_{2}$ es el logaritmo natural de la longitud o peso inicial y final. $\mathrm{N}_{\mathrm{o}}$ y $\mathrm{N}_{\mathrm{i}}$ es el número inicial y final de camarones sembrados.

\section{Calidad del agua.}

Los desechos sólidos acumulados en el fondo de los acuarios se extrajeron con sifón tres veces por semana. La calidad del agua se monitoreó cada siete días. Se determinaron oxígeno disuelto y temperatura mediante Oxímetro Hach $\left( \pm 0.01 \mathrm{mg} \mathrm{l}^{-1} ; \pm 0.01^{\circ} \mathrm{C}\right)$, salinidad con refractómetro VEE GEE A366ATC ( $\pm 0.5 \%$ ), y amonio total y nitritos con el test colorimétrico Nutrafin $\left( \pm 0.05 \mathrm{mg} \mathrm{l}^{-1}\right)$.

Análisis estadístico.

Se empleó el diseño estadístico completamente al azar. La normalidad de los datos se determinó mediante la prueba de Kolmogorov-Smirnov. Las diferencias entre las medias de los tratamientos se determinaron al 95\% por análisis de varianza y con la prueba de Tukey. Se utilizó el programa estadístico SPSS versión 23 para Windows.

\section{Resultados.}

Comportamiento: Los camarones expuestos en agua con $0 \%$ y $2 \%$ de salinidad mostraron fuerte interacción agresiva que ocasionó injurias, pérdida de los apéndices, y las muertes de los camarones fueron por canibalismo bien sea por efecto o no de la muda que sucedió principalmente en las noches porque, a primera hora de la mañana, se encontraron partes del cuerpo de los camarones dispersados en el acuario y también los exoesqueletos de aquellos camarones que mudaron. Los camarones sobrevivientes se desplazaron por el acuario y no consumieron todo el alimento suministrado. En cambio, aquellos camarones expuestos en agua con 4\% de salinidad, no se observó ningún tipo de interacción ni canibalismo, y la ración de alimento fue consumida completamente; pero la muerte de un camarón por repetición fue por causas desconocidas. En los acuarios se observaron algunas exuvias producto de mudas de los camarones.

Los camarones expuestos en agua con salinidad de $8 \%$, quedaron inmóviles al inicio del experimento y después de aproximadamente dos minutos se recuperaron, pero mostraron lenta actividad locomotora durante el experimento y solo consumieron parte de la ración suministrada. A los 32 días de experimentación se observó solo un exoesqueleto producto de la muda de un camarón. Los camarones muertos expuestos en agua con salinidad de $8 \%$ presentaron el cuerpo y los apéndices cefalotorácicos rígidos y el cuerpo fue de color marrón oscuro; no hubo otro signo más de muerte. No hubo muertes por canibalismo.

Supervivencia: Todos los camarones expuestos en agua con $4 \%$ de salinidad supervivieron hasta los 45 días, pero a los 60 días disminuyó a 80\% (12 camarones por tratamiento) y la supervivencia fue alta $y$ 


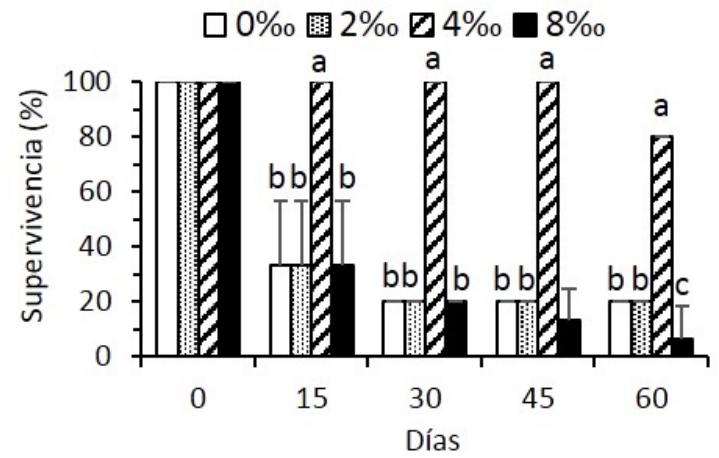

Figura 2. Supervivencia de machos adultos de $C$. caementarius expuestos a diferentes salinidades, durante 60 días. Letras diferentes sobre las barras en un mismo período indican que hay diferencia significativa $(\mathrm{p}<0.05)$ entre tratamientos.

significativa $(\mathrm{p}<0.05)$, desde los 15 días de experimentación, en relación con los demás tratamientos (Figura 2). Las muertes registradas en esta salinidad fueron por causas desconocidas.

En cambio, en agua con salinidades de $0 \%$, $2 \%$ y $8 \%$, la supervivencia de los camarones disminuyó abruptamente a 33\% (5 camarones) a los 15 días de iniciado el experimento y a 20\% (3 camarones) a los 30 días; pero a los 60 días solo aquellos expuestos en $8 \%$ disminuyó la supervivencia a $6 \%$ (un camarón de una repetición sobrevivió), siendo estadísticamente $(\mathrm{p}<0.05)$ diferente a los demás tratamientos (Figura 2). El $90 \%$ de las muertes de los camarones expuestos a $0 \%$ y $2 \%$ de salinidad se debió al canibalismo y el $10 \%$ a la muda y por causas desconocidas. En cambio, todas las muertes de los camarones expuestos a $8 \%$ de salinidad se debieron al estrés osmótico.

Crecimiento: El crecimiento en longitud de los camarones durante el período experimental fue similar ( $p>0.05$ ) entre tratamientos; con excepción de aquellos expuestos en agua con $8 \%$ de salinidad que aparentemente crecieron más desde los 15 días (Figura 3 ), pero ello, porque solo sobrevivieron tres o un solo camarón por repetición. Los parámetros de crecimiento

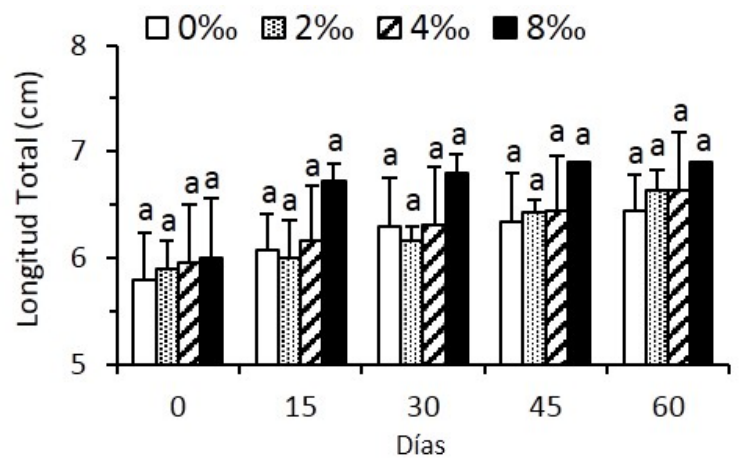

Figura 3. Crecimiento en longitud de machos adultos de $C$. caementarius expuestos a diferentes salinidades durante 60 días. en longitud de los camarones, al final del período experimental, no presentaron diferencias significativas $(\mathrm{p}>0.05)$ (Tabla 1).

El crecimiento en peso de los camarones durante el período experimental no presentó diferencia significativa $(p>0.05)$ entre tratamientos. Sin embargo, los camarones expuestos en agua con 4\%o de salinidad crecieron más desde los 30 días. En agua con $8 \%$ de salinidad los camarones aparentemente crecieron más desde los 15 días, porque solo sobrevivieron tres o un solo camarón por acuario; pero desde los 30 días, el peso húmedo de los camarones se estabilizó hasta el final del experimento (Figura 4). Los parámetros de crecimiento en peso de los camarones al final del período experimental no presentaron diferencias significativas $(p>0.05)$ entre tratamientos, pero hubo tendencia a incrementar en salinidad de 4\%o (Tabla 2).

Calidad el agua: Durante el experimento, no hubo diferencia significativa en los parámetros de la calidad del agua entre tratamientos (Tabla 3 ). El promedio de temperatura del agua fue de $25.22{ }^{\circ} \mathrm{C}$ y la concentración de oxígeno disuelto fue de $5.68 \mathrm{mg} \mathrm{l}^{-1}$. Los nitritos fluctuaron entre $0.10 \mathrm{mg} \mathrm{l}^{-1}$ y $0.31 \mathrm{mg} \mathrm{l}^{-1} \mathrm{y}$ el amonio total entre $0.02 \mathrm{mg} \mathrm{l}^{-1} \mathrm{y} 0.12 \mathrm{mg} \mathrm{l}^{-1}$.

\section{Discusión.}

Los camarones machos adultos de $C$. caementarius expuestos en $0 \%$ y $2 \%$ de salinidad mostraron fuerte interacción agresiva entre congéneres y hubo muertes por canibalismo desde el primer día de experimentación. Estos resultados evidencian, una vez más, la reputación agresiva de la especie principalmente en ejemplares machos en cultivo comunal y en agua dulce como ha sido informado en otros trabajos (Ponce, 1977; Rojas et al., 2012; Reyes, 2012), y además, la agresividad de la especie también se dio en agua de $2 \%$ de salinidad.

En agua con salinidad de 4\%o todos los camarones machos adultos de C. caementarius sobrevivieron durante 45 días de experimentación, y durante este período no hubo interacción ni canibalismo, aun cuando los camarones mudaron, siendo esta la primera

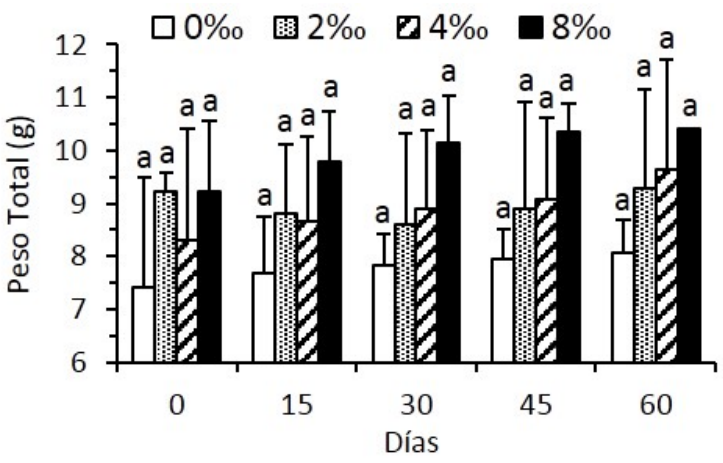

Figura 4. Crecimiento en peso de machos adultos de C. caementarius expuestos a diferentes salinidades durante 60 días. 
Ecol. apl. Vol. 16 № 2, pp. 75-82

Tabla 1. Parámetros de crecimiento en longitud de machos adultos de C. caementarius expuestos a diferentes salinidades durante 60 días.

\begin{tabular}{lcccc}
\hline \multirow{2}{*}{ Parámetros } & \multicolumn{4}{c}{ Salinidad } \\
\cline { 2 - 5 } & $0 \% 0$ & $2 \% 0$ & $4 \% 0$ & $8 \% 0$ \\
\hline LT inicial $(\mathrm{cm})$ & $5.80 \pm 0.44^{\mathrm{a}}$ & $5.90 \pm 0.26^{\mathrm{a}}$ & $5,96 \pm 0,55^{\mathrm{a}}$ & $6.00 \pm 0.56^{\mathrm{a}}$ \\
LT final $(\mathrm{cm})$ & $6.45 \pm 0.33^{\mathrm{a}}$ & $6.63 \pm 0.20^{\mathrm{a}}$ & $6,64 \pm 0,54^{\mathrm{a}}$ & $7.10^{*}$ \\
CA $(\mathrm{cm})$ & $0.65 \pm 0.10^{\mathrm{a}}$ & $0.73 \pm 0.07^{\mathrm{a}}$ & $0.69 \pm 0.037^{\mathrm{a}}$ & $1.11^{*}$ \\
GP $(\%)$ & $11.21 \pm 1.80^{\mathrm{a}}$ & $12.32 \pm 1.06^{\mathrm{a}}$ & $11.59 \pm 0.64^{\mathrm{a}}$ & $18.33^{*}$ \\
TCA final $\left(\mathrm{cm} \mathrm{dí}^{-1}\right)$ & $0.011 \pm 0.007^{\mathrm{a}}$ & $0.012 \pm 0.005^{\mathrm{a}}$ & $0.011 \pm 0.002^{\mathrm{a}}$ & $0.018^{*}$ \\
TCE final $\left(\% \mathrm{dia}^{-1}\right)$ & $0.177 \pm 0.117^{\mathrm{a}}$ & $0.194 \pm 0.069^{\mathrm{a}}$ & $0.182 \pm 0.041^{\mathrm{a}}$ & $0.281^{*}$ \\
\hline
\end{tabular}

* Dato de un solo camarón. LT: Longitud total. CA: Crecimiento absoluto. GP: Ganancia porcentual. TCA: Tasa crecimiento absoluto; TCE: Tasa de crecimiento específico. Datos con letras iguales en superíndices en una misma fila indica que no hay diferencia significativa $(\mathrm{p}>0.05)$.

evidencia de mantener, durante largo período, camarones adultos de la especie en este nivel de salinidad sin que suceda mortalidad, excepto cerca a los 60 días que murió solo un camarón por acuario pero por causas desconocidas. Estos resultados sustentan la hipótesis de que la salinidad del agua de 4\%, inhibiría el olor de los fluidos de la ecdisis del camarón por reacción con los iones del agua salobre, lo que no sucedió en salinidad de $2 \%$, probablemente por la baja concentración de los iones en el agua salobre. Según Chang (1992) los fluidos de la ecdisis están compuestos de aminoácidos, enzimas y de otras sustancias orgánicas del exoesqueleto viejo parcialmente digerido, que son estimulantes y atrayentes alimenticios muy fuertes para los crustáceos. Estos fluidos serían los que estimulan la interacción y el canibalismo en los ejemplares adultos, similar a lo informado en postlarvas de la misma especie cultivadas en agua con salinidades de $12 \%$ y $24 \%$, donde las sustancias liberadas durante la ecdisis son atenuadas por estas salinidades, con la consiguiente disminución de la interacción, el canibalismo y permitió supervivencias de $95 \%$ y $70 \%$, respectivamente (Reyes et al., 2006).

El crecimiento de machos adultos de $C$. caementarius en agua con salinidad de 4\%o no mostró diferencia significativa $(\mathrm{p}>0.05)$ con aquellos expuestos en agua con $0 \%$ y $2 \%$ de salinidad durante el período de estudio; pero en salinidad de 4\%o, los camarones ganaron mayor peso $(16 \%)$, consumieron todo el alimento proporcionado y no hubo interacción por alimento. Estos resultados sugieren que en los camarones adultos expuestos a salinidad de $4 \%$ se estimularía el metabolismo o la asimilación de nutrientes, como lo sucedido en Palaemonetes argentinus, expuesto en $8 \%$ de salinidad (Díaz et al., 2010).

Los resultados favorables del crecimiento en peso de $C$. caementarius expuesto en agua de $4 \%$ de salinidad, aunque sin diferencia significativa, sugieren que los organismos deben haber estado en equilibrio osmótico con el medio y por ello probablemente ganaron más biomasa. La hemolinfa de $M$. tenellum, se vuelve isosmótica en $10 \%$, pero los mejores resultados en crecimiento en peso se da en salinidades de 5\% y 10\%o (Vega-Villasante et al., 2011). En M. nipponense la hemolinfa se vuelve isosmótica a $15 \%$, pero mejor crecimiento se da en $14 \%$ de salinidad (Wang et al., 2004). Además, es probable que la exposición a salinidad de 4\% no solo favorezca el crecimiento de la especie sino más bien podría mejorar también la calidad del tejido muscular del camarón. En M. nipponense, cultivado en agua de $14 \%$ de salinidad, se mejora la concentración de proteínas y de aminoácidos libres en el músculo (Wang et al., 2004).

La lenta actividad locomotora y el bajo consumo de alimento, desde el inicio del experimento, que tuvieron los camarones machos adultos de $C$. caementarius expuestos en agua con $8 \%$ de salinidad, fue similar al

Tabla 2. Parámetros de crecimiento en peso de machos adultos de $C$. caementarius expuestos a diferentes salinidades durante 60 días.

\begin{tabular}{|c|c|c|c|c|}
\hline \multirow{2}{*}{ Parámetros } & \multicolumn{4}{|c|}{ salinidad } \\
\hline & $0 \%$ & $2 \%$ & $4 \%$ & $8 \%$ \\
\hline PT inicial $(\mathrm{g})$ & $7.40 \pm 2.08^{a}$ & $9.21 \pm 0.36^{\mathrm{a}}$ & $8.30 \pm 2.12^{\mathrm{a}}$ & $9.23 \pm 1.32^{\mathrm{a}}$ \\
\hline PT final $(\mathrm{g})$ & $8.07 \pm 0.61^{\mathrm{a}}$ & $9.28 \pm 1.86^{\mathrm{a}}$ & $9.63 \pm 2.10^{\mathrm{a}}$ & $10.90 *$ \\
\hline $\mathrm{CA}(\mathrm{g})$ & $0.67 \pm 0.78^{a}$ & $0.07 \pm 0.38^{\mathrm{a}}$ & $1.33 \pm 0.18^{\mathrm{a}}$ & $1.67 *$ \\
\hline GP $(\%)$ & $9.01 \pm 10.00^{\mathrm{a}}$ & $0.72 \pm 4.25^{\mathrm{a}}$ & $16.06 \pm 1.94^{\mathrm{a}}$ & $18.05^{*}$ \\
\hline TCA (g día $\left.{ }^{-1}\right)$ & $0.011 \pm 0.052^{\mathrm{a}}$ & $0.001 \pm 0.025^{\mathrm{a}}$ & $0.022 \pm 0.012^{\mathrm{a}}$ & $0.028 *$ \\
\hline TCE $\left(\% \mathrm{~g} \mathrm{día}^{-1}\right)$ & $0.144 \pm 0.643^{\mathrm{a}}$ & $0.012 \pm 0.284^{\mathrm{a}}$ & $0.248 \pm 0.124^{\mathrm{a}}$ & $0.277^{*}$ \\
\hline \multicolumn{5}{|c|}{$\begin{array}{l}\text { * Dato de un solo camarón. PT: Peso Total húmedo. CA: Crecimiento absoluto. GP: } \\
\text { Ganancia porcentual. TCA: Tasa crecimiento absoluto; TCE: Tasa de crecimiento } \\
\text { específico. Datos con letras iguales en superíndices en una misma fila indica que no } \\
\text { hay diferencia significativa }(\mathrm{p}>0.05) \text {. }\end{array}$} \\
\hline
\end{tabular}


Tabla 3. Parámetros físicos y químicos del agua de experimentación de machos adultos de C. caementarius expuestos a diferentes salinidades durante 60 días.

\begin{tabular}{lrrrr}
\hline \multirow{2}{*}{ Parámetros } & \multicolumn{4}{c}{ Salinidad } \\
\cline { 2 - 5 } & \multicolumn{1}{c}{$0 \% 0$} & \multicolumn{1}{c}{$2 \% 0$} & \multicolumn{1}{c}{$4 \% 0$} & \multicolumn{1}{c}{$8 \%$} \\
\hline Temperatura $\left({ }^{\circ} \mathrm{C}\right)$ & $25.30 \pm 0.00^{\mathrm{a}}$ & $25.20 \pm 0.10^{\mathrm{a}}$ & $25.30 \pm 0.20^{\mathrm{a}}$ & $25.10 \pm 0.10^{\mathrm{a}}$ \\
Oxígeno $\left(\mathrm{mg} \mathrm{l}^{-1}\right)$ & $5.67 \pm 0.03^{\mathrm{a}}$ & $5.62 \pm 0.08^{\mathrm{a}}$ & $5.73 \pm 0.10^{\mathrm{a}}$ & $5.71 \pm 0.22^{\mathrm{a}}$ \\
Nitritos $\left(\mathrm{mg} \mathrm{l}^{-1}\right)$ & $0.10 \pm 0.00^{\mathrm{a}}$ & $0.28 \pm 0.00^{\mathrm{a}}$ & $0.31 \pm 0.01^{\mathrm{a}}$ & $0.25 \pm 0.05^{\mathrm{a}}$ \\
Amonio total $\left(\mathrm{mg} \mathrm{l}^{-1}\right)$ & $0.04 \pm 0.03^{\mathrm{a}}$ & $0.12 \pm 0.07^{\mathrm{b}}$ & $0.08 \pm 0.03^{\mathrm{a}}$ & $0.02 \pm 0.00^{\mathrm{a}}$ \\
\hline
\end{tabular}

Datos con letras iguales en superíndices en una misma fila indica que no hay diferencia significativa $(\mathrm{p}>0.05)$.

comportamiento de $M$. acanthurus expuesto a $35 \%$ que pasaron a ser hipoconformadores y por ello tendrían alta permeabilidad en salinidades elevadas (Giareta, 2017), y también similar al de $M$. rosenbergii que reduce su actividad alimenticia en salinidad de $\geq 10 \%$ (Chand et al., 2015). Además, en $8 \%$ de salinidad, no hubo muertes por canibalismo, pero la mortalidad de los camarones C. caementarius con el cuerpo y los apéndices cefalotorácicos rígidos y el cambio de color del cuerpo a marrón oscuro, serían indicadores del funcionamiento de algún mecanismo adaptativo al estrés osmótico como la excreción de sal por transporte activo desde el epitelio branquial para reestablecer la homeostasis del medio interno (Freire et al., 2008; Sun et al., 2012) y el mantenimiento del volumen celular (Pinheiro, 2017), entre otros, que en algunos camarones de $C$. caementarius estos mecanismos no fueron eficientes porque murieron dentro de los primeros 15 días. Sin embargo, estos resultados son contrarios a lo registrado en $M$. rosenbergii, donde los adultos pierden habilidad para hiperregular a $17 \%$ de salinidad (Cheng et al., 2003). En P. argentinus, el incremento de la salinidad del agua ( $8 \%$ a $24 \%$ ) ocasiona reducción de la función metabólica y alteraciones citológicas en el hepatopáncreas (Díaz et al., 2010). En adultos de $M$. rosenbergii la mortalidad es asociada con la inhabilidad para mudar en agua con $25 \%$ de salinidad (Huong et al., 2010; Chand et al., 2015).

Los escasos camarones machos adultos de $C$. caementarius que sobrevivieron entre los 15 días y 60 días en agua con $8 \%$ de salinidad $(3,1$ y ningún camarón por repetición), sería indicador de la variabilidad del grado de resistencia al estrés osmótico que tendrían algunos organismos, probablemente por mecanismos fisiológicos o genéticos, que es necesario estudiar en la especie. En el caso de $M$. rosenbergii, cuando los organismos son expuestos a estrés salino de $10 \%$ y $24 \%$, se activan 10 genes conocidos y siete desconocidos localizados en la branquias; estos genes están involucrados en la regulación del metabolismo y mantenimiento de la homeostasis, en el proceso fisiológico de la eurihalinidad, en la osmorregulación, en la tolerancia al estrés salino, entre otros (Barman et al., 2012).

Los valores de crecimiento de los machos adultos de C. caementarius expuestos en agua con $8 \%$ de salinidad no son confiables toda vez que solo sobrevivió un camarón al final del experimento y como ya se explicó, el estrés osmótico afectó la supervivencia. Valdez et al. (2008) consideraron que las salinidades extremas para el camarón, origina un gasto energético en la osmorregulación a expensas de otros procesos, como el crecimiento. Así mismo, en altas salinidades el crecimiento de $M$. rosenbergii es afectado por el gasto energético, el ahorro de proteínas y agotamiento de reservas de lípidos, lo que a su vez afecta la biomasa (Chand et al., 2015).

La alta supervivencia ( $>80 \%$ ) y los mayores valores en el crecimiento de machos de C. caementarius expuestos en agua con $4 \%$ de salinidad, en relación con aquellos expuestos en $8 \%$, indican que la especie posee reducida capacidad fisiológica para soportar incrementos de salinidad del medio, lo cual es evidencia de que la especie es estenohalina. Estos resultados son contrarios a lo reportado en adultos de $M$. rosenbergii que muestra satisfactorio crecimiento y supervivencia, además de poseer fuerte capacidad hiperreguladora en agua dulce y en salinidades de hasta 15\%o (Cheng et al., 2003; Chand et al., 2015). Además, los resultados del estudio también sugieren que los camarones machos adultos de C. caementarius se encuentran, probablemente, más adaptados al medio dulceacuícola, similar a M. brasiliensis (Freire et al., 2003), pues mayor proporción de adultos se encuentran en las partes medias y altas del río (Yépez \& Bandín, 1998) y los camarones machos adultos de la especie evitan áreas estuarinas donde hay amplia variación de salinidad. Es conveniente estudiar las concentraciones iónicas de la hemolinfa y el punto isoomótico en $C$. caementarius que permita determinar la adaptación a bajas salinidades, de acuerdo a lo recomendado para camarones palaemónidos (Freire et al., 2003).

Los factores ambientales del agua de los acuarios se mantuvieron estables y estuvieron dentro de los valores registrados para los ríos de la costa peruana (Viacava et al., 1978; Yépez \& Bandín, 1998), con baja concentración de amonio $\left(<0.12 \mathrm{mg} \mathrm{l}^{-1}\right)$ y de nitritos $\left(<0.31 \mathrm{mg} \mathrm{1}^{-1}\right)$, según lo sugerido para especies dulceacuícola (New, 2002). Por lo que las muertes de machos adultos de C. caementarius expuestos en $0 \%$ y $2 \%$ de salinidad, se debieron al canibalismo; en cambio, en agua con $8 \%$ de salinidad, las muertes se debieron al estrés salino que la especie tuvo que soportar, como se explicó anteriormente, y más no a factores ambientales. Además, en adultos de A. scabra, se determina que si el gradiente osmótico sobrepasa el 
potencial regulatorio del organismo, el proceso de osmorregulación se interrumpe y el organismo muere (Rodríguez et al., 1991).

En el presente estudio se demostró por primera vez, a nivel experimental, la posibilidad de cultivo o mantenimiento de machos adultos del camarón de río C. caementarius, en agua salobre de baja salinidad (4\%o) donde se inhibe la interacción, el canibalismo y los camarones crecen más, aunque sin diferencias significativas. Estos resultados permitirán ser utilizados por aquellos cultivadores que dispongan de terrenos con baja concentración de salinidad. $M$. rosenbergii es cultivado en estanque de tierra y la mejor producción, mayor tamaño y alta supervivencia se da en agua de 5\%o que proviene de reservorios de irrigación (Nair \& Salin, 2006); así mismo, dicha especie muestra satisfactorio crecimiento y supervivencia en un amplio rango de salinidad $(0-15 \%)$ y tiene implicancias significativas para la selección de terrenos y en el mantenimiento de la salinidad del agua para maximizar la productividad comercial en áreas propensas a inundación costera (Chand et al., 2015). Además, como C. caementarius se comercializa entero y por peso, es recomendable el cultivo comunal en agua con salinidad de $4 \%$ para engorde hasta por 45 días. Así mismo, éste nivel de salinidad podría utilizarse también para el transporte de ejemplares vivos y para diferentes propósitos de investigación o de producción en cultivo intensivo.

\section{Conclusiones.}

Los camarones machos adultos de C. caementarius expuestos en agua con 4\% de salinidad presentaron alta $y$ significativa $(\mathrm{p}<0.05)$ supervivencia $(>80 \%)$, en cambio en $0 \%$ y $2 \%$ la supervivencia fue baja (20\%) debido al canibalismo y en $8 \%$ la supervivencia fue muy baja (6\%) debido al estrés osmótico.

El crecimiento de machos adultos de $C$. caementarius en agua con salinidad de 4\% no mostró diferencia significativa $(\mathrm{p}>0.05)$ con aquellos expuestos en agua dulce y en $2 \%$ durante el período de estudio, pero el mayor valor de crecimiento en peso húmedo se presentó en agua con $4 \%$ de salinidad.

\section{Literatura citada.}

Barman H.K, Patra S.K., Das V., Mohapatra S.D., Jayasankar P. Mohapatra C., Mohanta R., Panda R.P. \& Rath S.N. 2012. Identification and characterization of differentially expressed transcripts in the gills of freshwater prawn (Macrobrachium rosenbergii) under salt stress. The Scientific World Journal, Article ID 149361. doi:10.1100/2012/149361.

Bauer R.T. 2013. Amphidromy in shrimps: a life cycle between rivers and the sea. Lat. Am. J. Aquat. Res., 41(4): 633-650. DOI: 103856/vol41-issue4-fulltext-2.

Belli N.M., Faleiros R.O., Firmino K.C.S., Masui D.C., Leone F.A., McNamara J.C. \& Furriel R.P.M. 2009. $\mathrm{Na}, \mathrm{K}-\mathrm{ATPase}$ activity and epithelial interfaces in gills of the freshwater shrimp Macrobrachium amazonicum (Decapoda, Palaemonidae). Comparative Biochemistry and Physiology, Part A 152: 431-439.
Chand B.K., Trivedi R.K., Dubey S.K., Rout S.K., Beg M.M. \& Das U.K. 2015. Effect of salinity on survival and growth of giant freshwater prawn Macrobrachium rosenbergii (de Man). Aquaculture Reports. 2: 26-33.

Chang E.S. 1992. Endocrinology. pp. 53-93. En: Fast A.W. \& Lester L.J. (Eds). Marine shrimp culture: Principles and practices. Development in Aquaculture and Fisheries Science, 23. Elsevier Science Publisher B.V. The Netherlands.

Cheng W., Liu CH., Cheng CH. \& Chen J.C. 2003. Osmolality and ion balance in giant river prawn Macrobrachium rosenbergii subjected to changes in salinity: role of sex. Aquaculture Research. 34: 555.

Cornejo J., Pérez L. \& Reyes W. 2015. Efecto de la levadura Saccharomyces cerevisiae en la dieta de machos del camarón Cryphiops caementarius (Crustacea, Palaemonidae) sobre la cuenta total y diferencial de hemocitos. Revista Bio Ciencias. 3(3):176-186. http://dx.doi.org/10.15741/revbio.03.03.04.

Díaz A.C., Sousa L.G. \& Petriella A.M. 2010. Functional cytology of the hepatopancreas of Palaemonetes argentinus (Crustacea, Decapoda, Caridea) under osmotic stress. Braz. Arch. Biol. Technol. 53(3): 599608.

El-Sherif M. \& Ali A. 2009. Effect of rearing systems (monoand Poly-culture) on the performance of freshwater prawn (Macrobrachium rosenbergii) juveniles. Journal of Fisheries and Aquatic Science. 4(3): 117-128.

Freire C.A., Cavassin F., Rodrigues E.N., Torres A.H. \& McNamara J.C. 2003. Adaptive patterns of osmotic and ionic regulation, and the invasion of fresh water by the palaemonid shrimps. Comparative Biochemistry and Physiology. Part A 136: 771-778.

Freire C.A., Amado E.M., Souza L.R., Veiga M.P.T., Vitule J.R.S., Souza M.M. \& Prodocimo V. 2008. Muscle water control in crustaceans and fishes as a function of habitat, osmoregulatory capacity, and degree of euryhalinity. Comparative Biochemistry and Physiology, Part A. 149: 435-446.

Giareta E.P. 2017. O camarão de água doce Macrobrachium acanthurus Wiegmann, 1836 (Crustacea: Decapoda: Palaemonidae) e o desafio hiper-salino: expressão muscular de hsp70 e atividade da enzima branquial anidrase carbônica. (Dissertação Mestrado). Curitiba: Universidade Federal do Paraná. Brasil.

Guerra A., Gómez A., Velásquez E. \& Reyes W. 1987. Reproducción y crianza del camarón de río. Área Biomédica. 4(1): 899-915.

Huong D.T.T., Wang T., Bayley M. \& Phuong N.T. 2010. Osmoregulation, growth and moulting cycles of the giant freshwater prawn (Macrobrachium rosenbergii) at different salinities. Aquaculture Research. 41: 135-143.

Hill W.R. 2007. Fisiología animal comparada. Edit. Reverté, S.A. $248-260$ p..

Lleellish M., Silva I., Martínez C. \& Del Pozo P. 2005. Producto 4, Perú. Elaboración de criterios de cobertura geográfica para el establecimiento de áreas prioritarias para el desarrollo del Biocomercio. Elaborado por Grupo Técnico Producto 4 Perú. 90 p. Disponible: http://www.caf.com/attach/9/default/4CriteriosdeCobert uraGeográfica.pdf. 
Méndez M. 1981. Claves de identificación y distribución de los langostinos y camarones (Crustacea: Decapoda) del mar y ríos de la costa del Perú. Bol. Inst. Mar Perú. 5: 1170.

Moscoso V. 2012. Catálogo de crustáceos decápodos y estomatópodos del Perú. Bol. Inst. Mar Perú. 27(1-2): 1209.

Nair C.M. \& Salin K.R. 2006. Freshwater prawn farming in India. Status and prospects. Global Aquaculture Advocate. 9: 34-37.

New M.B. 2002. Farming freshwater prawns. A manual for the culture of the giant river prawn (Macrobrachium rosenbergii). FAO Fisheries Technical Paper. 428: 1-212.

Pezzato A.C. 1996. Balanceamiento de raciones para peces tropicales. Programa ALITE versión 1.10B.

Ponce J.E. 1997. Importancia del flujo de agua en los estanques - criaderos de camarón. Actas del Simposio sobre Acuicultura en América Latina. Documento de Investigación FAO, Informe de Pesca. 1: 240- 248.

PRODUCE (Ministerio de Producción). 2016. Anuario estadístico pesquero y acuícola 2015. Ministerio de la Producción, Perú. p. 29.

Reyes W. \& Lujan H. 2003. Estados y subestados del ciclo de muda del "camarón de río" Cryphiops caementarius (Molina, 1872) (Crustacea, Decapoda, Palaemonidae). En: II Congreso Iberoamericano Virtual de Acuicultura. CIVA 2003. 2003: 808-17. Disponible en URL: http://www.civa2003.org.

Reyes W., Bacilio S., Morales M. \& Mendoza R. 2006. Efecto de la salinidad en el crecimiento y supervivencia de postlarvas del camarón de río Cryphiops caementarius Molina, 1872 (Crustacea, Palaemonidae), en laboratorio. En IV Congreso Iberoamericano Virtual de Acuicultura. CIVA 2006. Disponible en URL: http://www.civa2006.org.

Reyes M. 2012. Crecimiento y supervivencia de adultos del camarón de río Cryphiops caementarius criados en sistema de recipientes individuales con recirculación de agua. (Tesis Doctoral). Trujillo: Universidad Nacional de Trujillo.

Rodríguez J.C., Marcano A.E. \& Marcano L.M. 1991. Tolerancia a la salinidad del camarón de río Atya scabra. Zootecnia Tropical. 9(2): 195-205.

Rojas R., Morales M.C., Rivadeneira M.M. \& Thiel M. 2012. Male morphotypes in the Andean river shrimp Cryphiops caementarius (Decapoda: Caridea): morphology, coloration and injuries. Journal of Zoology. 288(1): 2132.

Signoret G., Brailovsky P. \& Soto E. 1997. Comportamiento osmorregulador de Macrobrachium tenellum y Macrobrachium acanthurus (Decapoda:Palaemonidae) en diferentes salinidades. Revista Biología Tropical. 45: $1085-1091$

Sun M., Jiang K., Zhang F., Zhang D., Shen A., Jiang M., Shen X. \& Ma L. 2012. Effects of various salinities on

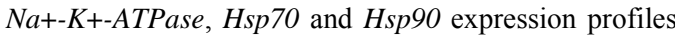
in juvenile mitten crabs, Eriocheir sinensis. Genetics and Molecular Research. 11(2): 978-986.

Valdez G., Díaz F., Re A.D. \& Sierra E. 2008. Efecto de la salinidad sobre la fisiología energética del camarón blanco Litopenaeus vannamei (Bone). Hidrobiológica. 18: 105-115.

Valverde O., Reyes W., Mendoza S. \& Yzásiga C. 2014. Hemocitos y oxihemocianina en hembras de Cryphiops caementarius Molina 1782 (Crustacea: Palaemonidae) criadas a diferentes salinidades. Revista AquaTIC. 40: 11-20.

Vega-Villasante F., Galavíz J.D., Guzmán-Arroyo M., Flores C.A. \& Espinosa-Chaurand L.D. 2011. Efecto de diferentes salinidades sobre el crecimiento y supervivencia de juveniles del langostino de río Macrobrachium tenellum (Smith, 1871). Zootecnia Tropical. 29(4): 467-473.

Viacava M., Aitken R. \& Llanos J. 1978. Estudio del camarón en el Perú. Bol. Inst. Mar del Perú. 3(5): 161-233.

Wang W.N., Wang A.L., Bao L., Wang J.P., Yuan L. \& Sun R.Y. 2004. Changes of protein-bound and free amino acids in the muscle of the freshwater prawn Macrobrachium nipponense in different salinities. Aquaculture. 233: 561-571.

Wasiw J. \& Yépez V. 2015. Evaluación poblacional del camarón Cryphiops caementarius en ríos de la costa sur del Perú. Rev Inv Vet Perú. 26(2): 166-181. http://dx.doi.org/10.15381/rivep.v26i2.11103.

Yépez V. \& Bandín R. 1998. Evaluación del recurso camarón de río Cryphiops caementarius en los ríos Ocoña, MajesCamaná y Tambo, Octubre 1997. Inf. Prog. Inst. Mar Perú, 77: 3-21. Disponible en: http://biblioimarpe.imarpe.gob.pe:8080/bitstream/handle /123456789/1259/IP\%2077.1.pdf?sequence=1.

\footnotetext{
${ }^{1}$ Egresado de la Escuela de Biología en Acuicultura. Facultad de Ciencias. Universidad Nacional del Santa. Av. Universitaria s/n. Urb. Bellamar, Nuevo Chimbote, Ancash (Perú). christion_14@hotmail.com.

${ }^{2}$ Egresado de la Escuela de Biología en Acuicultura. Facultad de Ciencias. Universidad Nacional del Santa. Av. Universitaria s/n. Urb. Bellamar, Nuevo Chimbote, Ancash (Perú). maxis1806@gmail.com.

${ }^{3}$ Laboratorio de Acuicultura Ornamental. Departamento de Biología, Microbiología y Biotecnología. Facultad de Ciencias. Universidad Nacional del Santa, Perú. Av. Universitaria s/n. Urb. Bellamar, Nuevo Chimbote, Ancash (Perú).wreyes_avalos@hotmail.com. Autor de correspondencia.
} 